\title{
Performance Analysis of Radio-Over-Fiber Communication System with Coherent Communication Technique
}

\author{
Hayder Hasan Khafeef ${ }^{1}$, Arvind Kumar Jaiswal ${ }^{2}$, Mukesh Kumar ${ }^{3}$ \\ ${ }^{1}$ M.Tech. in optical fibre Communication Engineering in Department of Electronics and Communication \\ Engineering in SHIATS, Allahabad, India. \\ ${ }^{2}$ Prof. and Head of ECE Dept at SHIATS-Allahabad, India. \\ ${ }^{3}$ Asst. Prof. in the Department of Electronics \& Communication Engineering in SHIATS, Allahabad, India.
}

\begin{abstract}
Radio over Fiber technology (RoF), an integration of wireless and fiber optic networks, is an essential technologyfor the provision of untethered access to broadband wirelesscommunications in a range of applications including last milesolutions, extension of existing radio coverage and capacity, and backhaul.Fullduplex radio-over-fiber (ROF) transport systememploying broadband nonlinear distortions suppressionscheme is proposed and demonstrated. Data rate of $10 \mathrm{GHz} / 90 \mathrm{Mbps}$ signal is externally modulated andtransmitted longhaul fiber link. A data streamof 90 Mbps transmitted over an 70-km single-mode fiber(SMF) transmission both for down/up-link with good biterror rate (BER) performance was achieved.
\end{abstract}

\section{Introduction}

The microwave/millimeter-wave ROF transportsystems, which integrate the advantages of wireless radioand fiber optical communications, have been developedwith high expectations for future communications thatrequire ultra-high-speed, higher capacity, and lower cost[1], [2].In such way, systems' bandwidth suffers fromthe limitation of RF devices' characteristic. And further,expensive RF devices will increase capital expenditures.So that a successful deployment of ROF transportsystems strongly depends on the availability of simplearchitecture. In this paper, a full-duplex ROF transportsystem based on direct-detection scheme is proposed anddemonstrated. With the assistance of light injectiontechnique at the transmitting site and optical band-passfilter (OBPF) at the receiving site, the optical carrier andone of the sidebands are eliminated before detecting.Only one optical sideband is processed by opticaldevices, and the digital baseband signal is obtaineddirectly from the sideband.

Electrical generated RFsignal comes from the beating between two opticalwavelengths after PD detection, and the carrierfrequency of the electrical generated RF signal is thesame as the frequency difference between these twooptical wavelengths [3], [4]. Thereby, the electrical RF signal cannot be obtained from only one opticalsideband without optical carrier and the other opticalsideband. The generated signal should be digitalbaseband signal. In our proposed approach, the RFpower degradation can be avoided even when the opticalcarrier is transmitted. A data stream of $90 \mathrm{Mbps}$ transmitted over an 70-km single-mode fiber (SMF)transmission both for down/up-link with low bit errorrate (BER) values were obtained.

Wireless transmission networks have been demandedfor different kinds of multimedia services. To meet theincreasing demands, the high-speed optical accessnetworks should be integrated with the flexibility ofwireless ones. Radio-over-fiber (ROF) transport systems,the integration of optical and wireless access networks,have potentially provided flexibility and large capacity[5-7]. For a practical implementation of fullduplex ROFtransport systems for multiple wavelengths transmission, the simplification of light source and the suppression ofnonlinear distortions are the key issues to be solved. Forlong-haul lightwave transmission, nonlinear distortionstake a vital role to degrade the performance of systems.In order to improve the performance of systems, it isnecessary to use some schemes to mitigate the nonlineardistortions.In this paper, a potentially cost-effective fullduplex of transport system based on nonlinear distortions suppressionscheme is proposed and demonstrated. A data rate of $10 \mathrm{GHz} / 70 \mathrm{Mbps}$ signal is externally modulated andtransmitted over a long-haul fiber link. Low bit error rate(BER) value and clear eye diagram were obtained in ourproposed systems.

\subsection{Basic ROF architecture}

\section{System Design Model}

Today RoFsystems, are designed to perform addedradio system functionalities besides transportation andmobility functions. These functions include datamodulation, signal processing, and frequency conversion(up and down). For a multifunctional RoF system, therequired radio signal at the input of the RoF systemdepends on the RoF technology and the functionalitydesired. Figure 1.shows a typical RF signal (modulated byanalog or digital modulation techniques) being transportedby an analog fiber optic link. The RF signal may be 
baseband data, modulated IF, or the actual modulated RFsignal to be distributed. The RF signal is used to modulatethe optical source in transmitter. The resulting opticalsignal is launched into an optical fiber. At the other end ofthe fiber, we need an optical receiver that converts theoptical signal to RF again. The generated electrical signalmust meet the specifications required by the wirelessapplication be it GSM, UMTS, wireless LAN, WiMax orother. By delivering the radio signals directly, theoptical fiber link avoids the necessity to generate highfrequency radio carriers at the antenna site. Since antennasites are usually remote from easy access, there is a lot

to gain from such an arrangement. Usually a single fibercan carry information in one direction only (simplex)which means that we usually require two fibers for bidirectional(duplex) communication.

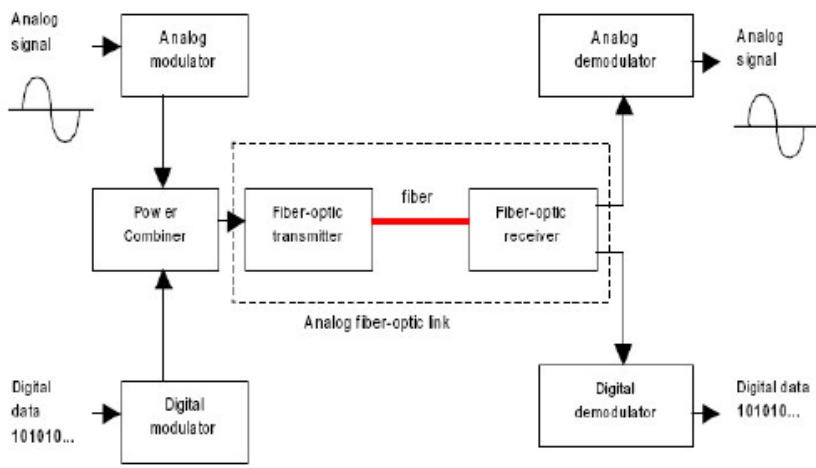

Figure 1. Basic ROF architecture

\subsection{Experimental setup}

The experimental configuration of our proposed full-duplexROF transport systems employing broadbandASE light source and nonlinear distortions suppressionscheme is shown in Fig 2.For down-link transmission, the central station (CS)is composed of a broadband ASE light source, twoEDFAs, a MachZehnder modulator (MZM), amicrowave signal generator, and a pair of AWGmultiplexer (MUX)/DEMUX. Four wavelengths of $\lambda 1$ (channel 1), $\lambda 2$ (channel 3), $\lambda 3$ (channel 5), and $\lambda 4$ (channel 7) from the odd channels of AWG DEMUXoutput were selected for down/up-link light sources. 90-Mbps data stream is mixed with microwave carrier $(10 \mathrm{GHz})$ to generate the compatible WiMAX data signal, and the resulting microwave data signal is supplied to the MZM.

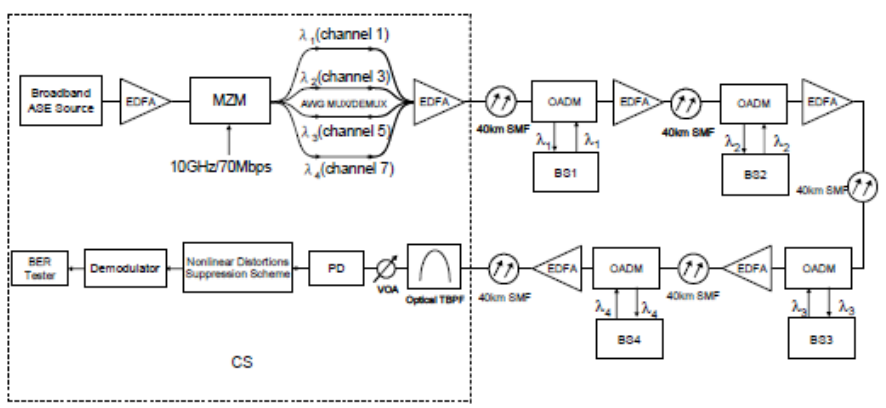

Fig.2. Experimental configuration of our proposed full-duplex ROF transport systems.

Signal is generated at the CS and then distributed tothe remote base stations (BSs) by using cascaded EDFAs.Each BS is addressed by individual wavelength for anoptical add-drop multiplexer (OADM). When many BSsare deployed in fiber networks, all down-linkwavelengths are employed within the wavelength rangeof 1530-1560 nm. The optimum modulationperformance will be achieved because the 12-GHz bandMZM employed in this work exhibits an optimummodulation performance from 1530 to $1560 \mathrm{~nm}$. Thefull-duplex ROF transport systems exploit the availablebandwidth of 1530-1560 $\mathrm{nm}$ to address multiple BSs.

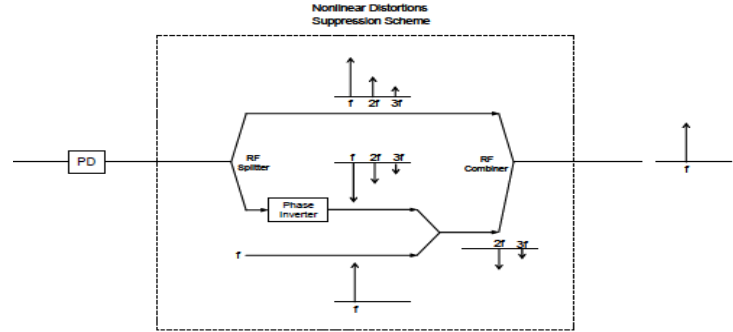

Fig. 3. Functional diagram of the nonlinear distortionssuppression scheme. 
The down-link data signal is adjusted by a variableoptical attenuator (VOA), detected by a broadbandphotodiode (PD), passed through a nonlinear distortionssuppression scheme, demodulated, and fed into a BERtester for BER analysis at each BS. The up-link datasignal is added to the fiber backbone and transmitted tothe CS, where they are separated using an optical tunableband-pass filter (TBPF) to select the desired wavelength.The signal is adjusted by a VOA, detected by a PD,passed through a nonlinear distortions suppressionscheme, and also fed into a BER tester for BER analysisafter demodulation.

\section{Simulation Results}

A functional diagram of the nonlinear distortionssuppression scheme is illustrated in Figures.

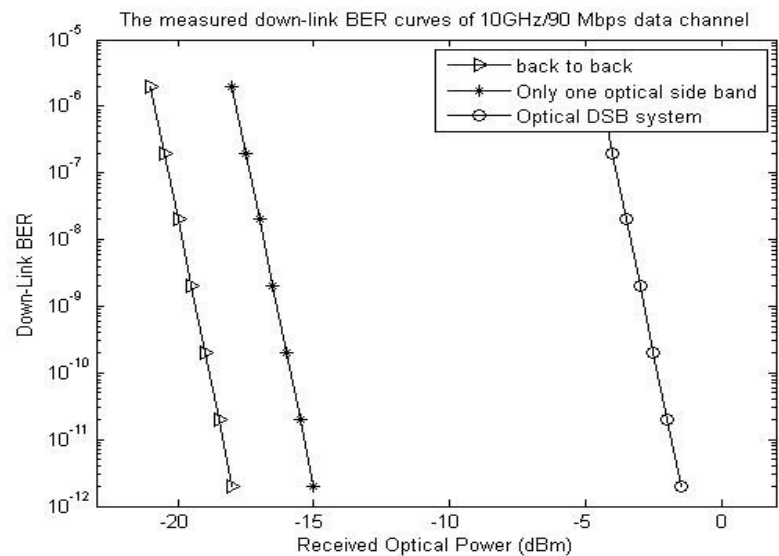

Nonlinearitycauses second-order and third-order harmonic (2HD and3HD) of the carrier to appear in the output intensityspectrum. The function of the nonlinear distortionssuppression scheme is to suppress the undesired $2 \mathrm{HDand} 3 \mathrm{HD}$, ideally leaving only the carrier at the output.

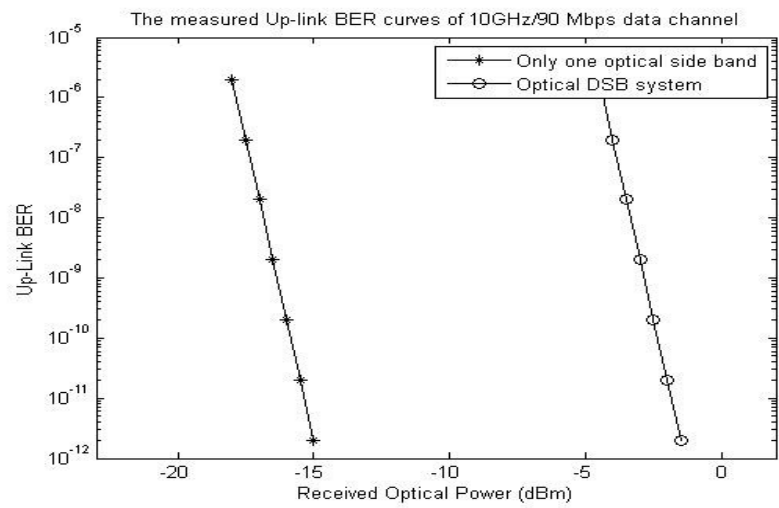

After the optical signal detected by the PD, the output of the PD is separated off by a $1 \times 2 \mathrm{RF}$ splitter, one of theoutput is applied to the phase inverter. The outputproducts at the phase inverter output are $180 \mathrm{o}$ phaseinversion, then combined with the stored copy of carrierto create $2 \mathrm{HD}$ and $3 \mathrm{HD}$ with $180 \mathrm{o}$ phase inversion.

\section{Conclusion}

We have proposed a full-duplex ROF transportsystem employing broadband nonlinear distortions suppression scheme to suppressnonlinear distortions like 2HD and 3HD. The feasibilityof our proposed systems is demonstrated andaccompanied with good BER performance and clear eyediagram over a long-haul fiber link.

\section{References}

[1]. H. H. Lu, H. L. Ma, and A. S. Patra, "Radio-on-fibertransport systems integration with 622 Mbps basebandtransmission," IEEE Photon. Technol. Lett., vol. 20, pp. 1618-1620, 2008

[2]. C. T. Lin, J. Chen, P. C. Peng, C. F. Peng, W. R. Peng, B. S.Chiou, and S. Chi, "Hybrid optical access network integratingfiber-tothe-home and radio-over-fiber systems," IEEE Photon.Technol. Lett., vol. 19, pp. 610-612, 2007.

[3]. Q. Chang, H. Fu, and Y. Su, "Simultaneous generation andtransmission of downstream multiband signals and upstreamdata in a bidirectional radio-over-fiber system," IEEE Photon. Technol. Lett., vol. 20, pp. 181-183, 2008.

[4]. Z. Jia, J. Yu, D. Boivin, M. Haris, and G. K. Chang,"Bidirectional ROF links using optically up-converted DPSKfor downstream and remodulated OOK for upstream," IEEEPhoton. Technol. Lett., vol. 19, pp. 653-655, 2007.

[5]. Serdyuk, V. M., "Dielectric study of bound water in grain atradio and microwave frequencies," Progress InElectromagnetics Research, PIER 84, 379-406, 2008 . 
[6]. Oraizi, H. and S. Hosseinzadeh, "A novel marchingalgorithm for radio wave propagation modeling over roughsurfaces," Progress In Electromagnetics Research, PIER 57,85-100, 2006.

[7]. Lu, H. H., A. S. Patra, W. J. Ho, P. C. Lai, and M. H. Shiu, “A full-duplex radio-over-fiber transport system based on FPlaser diode with OBPF and optical circulator with fiber Bragggrating," IEEE Photon. Technol. Lett., Vol. 20, 1652-1654,2007.

\section{Author's ProfiLe}

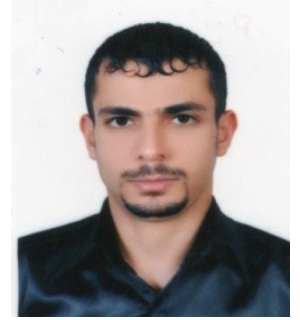

\section{Hayder Hasan Khafeef}

Received his bachelor of communication engineering from Technical College Najaf in IRAQ. M.Tech. in optical fibre Communication Engineering in Department of Electronics and Communication Engineering in SHIATS,Allahabad in India. Email: haider,alumary@yahoo.com

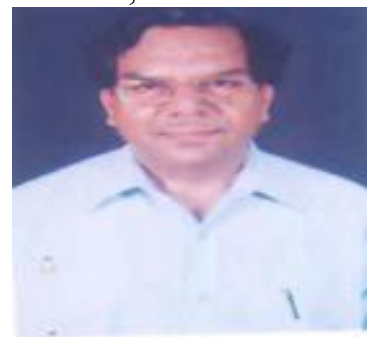

A.K. Jaiswal is Prof. and Head of ECE Dept at SHIATS-Allahabad. He Obtained M.Sc. in Tech. Electronic \& adio Engg. from Allahabad University in1967. He guided various projects \& research at undergraduate \& postgraduate level. He has more than 35 years Industrial, research \& Teaching experience and actively involved in research and publications. His area of interest includes Optical Networks and satellite communication.

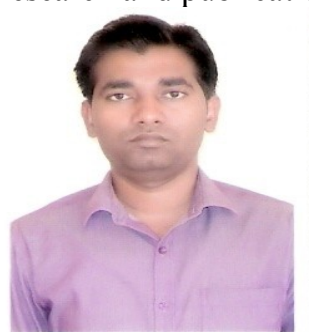

Mukesh Kumar is working as a Asst. Prof. in the Department of Electronics \& Communication Engineering in SHIATS, Allahabad. He received his M.Tech. Degree in Advanced Communication System Engineering from SHIATS, Allahabad in 2010. His research is focused on Signal processing, Wireless Sensor Network and Computer Networks ,Microwave Engineering, as well as Optical fiber communication 